\title{
ZU CODEX JUN. XI.
}

Bei den verhandlungen über die herkunf̣t der im codex Jun. 11 enthaltenen sammlung angelsächsischer gedichte ist ein punkt bisher unberücksichtigt gelassen worden, welcher in dieser frage vielleicht einiges licht verbreiten kann. Ich meine die beobachtung von Rieger, verskunst s.12, anm., dass in der Genesis und Exodus ea bisweilen mit $z$ alliteriert. Unter hinweglassung des zweifelbaft uberlieferten verses Exod. 288, sind die beispiele folgende:

zeorne tôzeánes, and sર̂̉on ealles panc - Gen. B 238

pâ wæs iû zêre ealdan wîtum - Ex. 33

wêron i[u]nze men ealle ætzædere - Ex. 190

eád and æð̀elo: hê wæs zearu swâ peáh - Ex. 339.

$\mathrm{Zu}$ diesen komnt, wie ich glaube, auch noch der vers iu âhte ic zeweald ealles wundres - Satan 107,

da in demselben die alliteration gewiss auf ia, nicht auf ahte ruhte.

Rieger bemerkt zur erklärung dieser erscheinung: 'ein solches verfahren setzt bei dem betreffenden dichter fur den anlaut $g$ in allen fällen die aussprache $j$ voraus, der auch die vocalische alliteration, besonders die auf $e a$, eo, nicht versagt zu werden brauchte.' Ich weiss nicht, ob Rieger mit der zuletzt gemachten einschränkung selbst auf diejenige deutung hinweisen will, die mir die einzig mögliche zu sein scheint, nämlich die, dem ea, eo die aussprache jea, jeo oder $j a$, jo zuzuschreiben, uber die ich zuletzt Beitr. IX, $208 \mathrm{f}$. gehandelt habe (ich verweise dazu nachträglich auf die damals von mir ubersehenen erörterungen Zupitza's im anz. f. deutsches altert. II, $5 \mathrm{f}$.). Lesen wir in den angeführten zeilen zealle $(s)$, zealdan, zeád, so entsprechen diese formen genau den am a.a.o. band- 
schriftlich nachgewiesenen zearfoðe, unzeáðe etc. für earfoðe, uneáde.

Wenn dies richtig ist, so ist anglischer, speciell northumbrischer, ursprung fur Genesis B, Exodus und Satan I ausgeschlossen, denn auf dem ganzen anglischen gebiete lauten eall, eald bekanntlich all, ald, genauer all, âld mit dehnung, und daraus konnte sich naturlich kein zeall etc. entwickeln.

Hierzu stimmt vortrefflich eine weitere dialektische eigenheit wenigstens der Genesis B, nämlich die behandlung der 2. 3. sing. ind. der (langsilbigen) verba und der participia praeteriti schwacher verba auf dentale. Wie Beitr. IX, $173 \mathrm{f}$. ausgeführt ist, finden sich hier synkopierte formen in der prosa nur im süden, auf sächsischem und kentischem boden. In der poesie fehlen sie, ausser in den Metris (wie bereits angemerkt), in dem Menologium, und in der Genesis B, ganz vereinzelte ausnahmen abgerechnet, deren vorhandensein ich nicht leugnen möchte, da ich nicht alle texte wieder auf diese frage hin durchgepruft habe. In Gen. B finden wir wyrd 236. 431. 552. 558, weorठ 405. 519 (cymठ 315. 806), āhwel 406, sent 515, zetrŷnঠ 569 (cwyঠ 581), lêcst 619 (forzifठ 662, lizst 734), slît 802, blîcঠ 811, an participien zesett 252, forlêed(d) 598. 630, zelêst 727, und von diesen sind eine ganze anzahl, wie ich demnächst nachweisen werde, metrisch als synkopiert sichergestellt. Für die anderen in frage kommenden gedichte lässt dies kriterium freilich im stich, aber nur deshalb, weil das in betracht kommende material zu gering ist; in der ganzen Exodus finden sich z. b. uberhaupt nur drei 3. personen sing. ind., die hierher fallen könnten, wyrceð 282, dêmeð 542, lêedeð 543 ; bei solchen geringen zahlen ist man kaum zur erwartung von ausnahmen berechtigt. Aber auch nach dem erstgenannten kriterium allein bleibt ja nur der suden des landes als heimat der besprochenen gedichte ubrig, und selbst hier lassen sich die grenzen noch enger ziehen. Ich glaube, die ganze sammlung ist in Ként oder dessen nachbarschaft zusammengebracht worden, für einzelne der gedichte auch direkt kentischer ursprung nachzuweisen.

Zunächst erinnern jene zea für $e a$ natürlich an die vielbesprochenen mittelkentischen yea, ya (zur literatur s. Danker, Die laut- und flexionslehre der mittelkent. denkmäler, Strass- 
burg 1879, s. 7 ff.); denn dass wirklich altes $e a$ im kentischen zu jea orler $j a$ werden konnte, geht meines bedankens trotz aller einwände aus der grleichung altkent. Ealdinz nom. loci Cod. dipl. II, $260=$ neukent. I'aldiny hervor. Hierzu stimmt, dass die angefuhrten unzeúde, zearfoðe sich im Boethius finden; denn beide handschriften dieses textes gehen (wie demnächst von anderer seite ausfihhrlicher wird dargelegt werden) auf ein kentisches original zurïck '). Nach Kent weist aber mit grosser wahrscheinlichkeit auch noch eine andere sehr charakteristische form, nämlich styde mit seinem rätselhaften $y$ fur stede locus. Die form begegnet in der handschrift viermal, zlêdstyde Gen. 1810, folcstyde Gen. 2000, und mit nachträglicher correctur zu stede in styde Gen. 356 und burhstyde Satan 363. In den tibrigen poetischen texten erscheint die form nicht mehr; dagegen ist $y$ der ausschliesslich bezeugte vocal dieses wortes im northumbrischen, wo dasselbe styd lautet (z. b. in Lind. Matth. p. 11,16. 12, 5, cap. 26, 52. 27, 32. 28, 26, Marc. 1, 35, Job. 10, 40, stydd Luc. 10,1; dat. on styde Rit. 101, $1^{\mathrm{b}}$, vgl. auch stydfastnise Rit. 20,1. 2, vnstydfullv Rit. 121, 1, vnstydfullnisse Rit. 192,1; im Ps. und Rushw. ${ }^{1}$ fehlt das wort; in Rushw.2 steht steyde Me. 1, 35, was wie eine correctur von stede zu styde aussieht). Ausserdem findet sich aber die form styde mehrmals in ortsnamen und zwar fast ausschliess-

1) Ich verweise hier der kïrze halber nur anf die zahlreichen $e$ fïr $y$, die in den Metra erscheinen, wie wercest 4, 18, wercan 26, 73, unnet 10,17 . 16, (i. 22, 10. 17 , unnetta 25,44 , unnetne 28,52 , zeheded 20,151 , semie 20,195, unmendlinza 25,30, ymbhwerft 28,12, rene 29, 10 für nyriest, wyrcan, unnyt, zchŷded, symle, unmyndlinga, ymbhwyrft, ryne. Ueb:igens liegt es nahe, auch die verse

Jôbes eafera se wæs zeó cyning 26,35

ealde ze ziunze ealle forhwerfde 26,86

hierlerzuziehen, indem man den ersten halbversen doppelalliteration gibt. Dask sich das tibrigens nicht mit der - an sich höchst unwahrscheinlichen - annahme verträgt, Aelfred habe auch den versificierten text der Hetra verfasst, liegt auf der hand. Ich glaube auch nicht an die verfisserschaft des künigs, sondern bin der meinung, dass die sog. Metra in kintischer mundarr, also doch wol von einem Kenter, auf grund einer berets in kentischen dialekt umgesetzten handschrift des ganzen prosatextes gearbeitet worden sind. Unsere beiden handschriften bieten freilich nicht mehr ganz den alten kentischen text, sondern eine unvollkomnrne riickübertragung in den sächsischen dialekt. 
lich in Kent und den angrenzenden grafschaften. Die ältesten belege stehen in der in kentischer mundart abgefassten urkunde des königs Aethelberht von Wessex rom jahre 862, Cod. dipl. II, 72 ff. no. 287 = Sweet, 0. E. T. 438 f.: tô Bipplestydae . Janne fram Bipplestyde tô Acuslyde ... fram Acustyde; sämmtliche angefuhrten ortschaften liegen in der nähe von Bromley in Kent (Fearnbiorzinza mearc z. b. ist Farnborough, SO. von Bromley; Acustyde wird von Kemble mit Oxted in Surrey identificiert). In der widerholung dieser urkunde durch Aethelred vom jahre 987, Cod. dipl. III, 226 ff., erscheinen die namen ebenfalls in der gestalt Bipplestyde und Acustyde, nur bei der widerholung des letzteren ist in sächsischer form Acustede geschrieben. Demnächst bietet die (nur in alter abschrift vorliegende) fälschlich vom jahre 790 datierte urkunde desselben königs, welche von Kemble, Cod. dipl. II, 70 in die jahre 860 bis 862 gesetzt wird, ebenfalls in Kent, stidlich vom Medway, den namen Horsūstyde. Aus Surrey liefert die urkunde des dux Aelfred (zwischen 871-889), Cod. dipl. II, 120 no. $317=$ Sweet, 0. E. T. $451 \mathrm{ff}$. die fromen Sondemstyde, Netelamstyde und Netelhamstyde neben einmaligem Sondenstede. Hiermit sind die alten belege erschöpft. Weitere verbreitung der form styde in den klustengebieten im norden von Kent bezeugen jedoch noch die beiden nur in späten, 'halbsächsischen' ab. schriften' erhaltenen urkunden Cod. dipl. III, $271 \mathrm{ff}$. no. 685 und IV, 208 no. 853; die erstere nennt Granstydae neben Grenstede = Greenstead in Essex, und Polstydie neben Polstede= Polstead in Suffolk, die letztere Rinzstyde = Ringstead in Norfolk (daneben auch on êlce und on ĥ́frîce styde).

Ausser diesen dem sudosten Englands entstammenden belegen habe ich nur noch ein styde in weit abliegender gegend gefunden. Das dem 11. jahrh. entstammende cartular von Worcester, cod. Cotton. 'l'ib. A. XIII, uberliefert nämlich in einer 814 ausgestellten schenkungsurkunde des königs Kenulf von Mercia die form Dunhamstyde, Cod. dipl. I, 255 (und III, 389) no. 202. Die lage dieses ortes ist aus dieser urkunde nicht zu bestimmen; aber in einer andern urkunde derselben sammlung, Cod. dipl. III, 259 f. no. 680, verleiht erzbischof Oswald von York, der vormalige bischof von Worcester, unter einstimmung tas hêredes at Wiozernaceastre, eine hide land 
at Hymeltane, bei deren grenzbestimmungen unter anderem auch Dûnhếmstedes acerzeard genannt wird, und da Hymeltân - Himbleton in Worcester nach Kemble feststeht, ist auch Dìnhâmstede in jener grafschaft zu suchen. Wir hätten somit einen beleg fur mercisches styde, welches passend dem northumbrischen styd zur seite träte. Für die ortsbestimmung unserer gedichtsammlung kann aber dieses styde so wenig in betracht kommen, wie die northumbrischen slyd; denn Dunhamstyde liegt auf einem boden, wo wir kein zeall erwarten können. Styde und zea für ea $\mathrm{z}$ us a m men weisen wie ich glaube bestimmt auf Kent und nachbarschaft hin, und dort, in Kent oder Surrey, wird denn auch mit Groschopp, Anglia VI, 265 ff. die quelle der eigentümlichen dialektformen zu suchen sein, welche die handschrift des sog. Satan auszeichnen. Doch dartiber lässt sich einstweilen nicht discutieren, ehe eingehendere untersuchungen wenigstens den versuch gemacht haben zu zeigen, was in den gemeinhin fur kentisch angesehenen texten und urkunden wirklich als charakteristisch fur diesen dialekt betrachtet werden darf.

TÜBINGEN, 17. april 1884.

\section{NOTIZEN ZU THOMAS BIRCK.}

Ausser den beiden kürzlich von H. Holstein in der Zs. f. deutsche philologie XVII, $71 \mathrm{ff}$. ausfuihrlich besprochenen dramen, der Comoedia gegen die doppelspieler und dem Ehespiegel, hat Thomas Birck noch ein drittes spiel verfasst, einen Hexens)iegel, der aber nicht zur veröffentlichung gelangt ist. Er selbst hat uber die schicksale dieses seines letzten dichtwerkes in der ausfibrlichen vorrede $\mathrm{zu}$ der Letze Predig berichtet, mt der er sich bei seinem abgang von Unterturkheim von seinen alten pfarrkindern verabschiedete. Exemplare dieses bisher ziemlich unbekannt gebliebenen ${ }^{1}$ ) opus befinden sich auf

1) L. M. Fischlin, Memoria theologorum Würtembergensium I, $294 \mathrm{f}$. ciiert die schrift, die er offenbar nicht selbst gesehen, unter dem ungenauen titel 'Valet-Predigt auss Act. XX.' und gibt fälschlich Tubingen stitt Speyer als druckort an. 